\title{
Measurement and modeling of kinematic error and clearance in harmonic drives
}

\author{
Chuang Zou ${ }^{\mathrm{a}}$, Tao Tao ${ }^{\mathrm{b}}$, Gedong Jiang ${ }^{\mathrm{c}}$, Pengfei Zeng ${ }^{\mathrm{d}}$ and Hongyang Du \\ School of Mechanical Engineering, Xi'an Jiaotong University, Xi'an, China \\ a zouchuang156@sina.com, b taotao@mail.xjtu.edu.cn, c gdjiang@yeah.net, \\ d1552695066@qq.com, ${ }^{\mathrm{d}} 714177551 @ q q . c o m$
}

\begin{abstract}
Keywords: harmonic drive; kinematic error; clearance; measurement; model
Abstract. In order to obtain the kinematic error and clearance in harmonic drive, an experimental setup was established. The cause of beat frequency characteristic was analyzed and verified by the FFT result of experiment data. The position dependent kinematic error was modeled by Fourier expression method and the simulated model was also verified by another set of experimental data. And a reversing experiment was designed to acquire the change process of clearance in harmonic drive and the frequency domain method was applied to extract the clearance value. The proposed measurement and modeling method will be useful to the dynamic modeling and performance evaluation of harmonic drive.
\end{abstract}

\section{Introduction}

Harmonic drive was invented in 1950s based on the elastic theory for the requirement of aerospace. As it has the characteristics of large ratio, high accuracy, small clearance, high load capacity and small volume and weight, harmonic drive has been widely used in satellite, industrial robot, missile, laser instrument and various kinds of measurement devices [1].

Harmonic drive is composed of three components which are wave generator, flexspline and circular spline as shown in Fig.1. The harmonic drive can be a reducer or an increaser when one of its three components is fixed. The harmonic drive is often set as a reducer that its wave generator (input) forces the gear teeth on flexspline into the fixed circular spline and then the flexspline (output) rotates in opposite direction. As the flexspline only rotates for the angle of two or three teeth while the wave generator rotates for a whole circle, the harmonic drive can realize large ratio of 30 160.

As harmonic drive is a kind of mechanism composed of three key components, the kinematic error caused by its manufacturing and assembling error among these components is responsible of its performance gradation. Both Good et al. [2] and Hidaka et al. [3] found the harmonic drive attributes' effect on system dynamics. Tuttle [4] noticed the kinematic inaccuracy of harmonic drive is one of the reasons for the systems problematic dynamic behaviors in the operating velocity measurement. The measurement and modeling of the kinematic error are of great significance to recognize the whole dynamic behavior of the harmonic drive. Most researchers [4-7] have measured the kinematic error and modeled it by using trigonometric functions. Ghorbel [5] proposed that the pure kinematic error must be obtained through careful experiment at low, uniform driving velocities because it is only caused by manufacturing or assembling error instead of any elastic component. Although the cause of kinematic errors is also explained in his paper, but the beat frequency characteristic was not noticed and analyzed. The clearance property is also one kind of important characteristic. Although the clearance of harmonic drive is very small, the gap in aligning coupling or wave generator will cause unavoidable clearance. It is difficult to measure and model the clearance of harmonic drive as it is blended with the kinematic error and difficult to be dissociated. And seldom researcher has discussed the clearance measurement in harmonic drive.

So, the established experimental setup and measurement method will be introduced firstly in this paper, and then the beat frequency characteristic of kinematic error in harmonic will be analyzed, the process of modeling of the position dependent kinematic error will be represented, and last the measurement and extraction method of the clearance in harmonic drive will be discussed. 


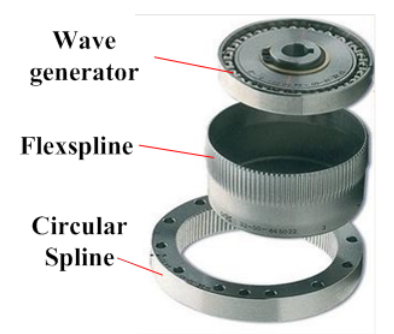

Fig.1 Structure of harmonic drive.

\section{Experimental setup and measurement}

Measurement method. Kinematic error in harmonic drive is defined as the deviation between the actual output rotation and the expected output rotation and could be represented by the following equation.

$$
\hat{\varphi}=\varphi_{o}-\frac{\varphi_{i}}{i}
$$

where $\hat{\varphi}$ is the kinematic error value, $\varphi_{i}$ is the actual input rotation of the harmonic drive and it becomes expected output rotation after it is divided by the transmission ratio $i, \varphi_{o}$ is the actual output rotation. As the manufacturing errors and assembling errors are the key factors of the kinematic error occurrence in harmonic drive transmission, the kinematic error have to be measured through low velocity experiment to avoid other flexibility induced factors. The input rotation and the output rotation should be gathered and saved synchronously when the input motor is driven at a low uniform velocity to acquire the kinematic error according to Eq.1.

Experimental setup. An experimental setup was established to measure the kinematic error in a harmonic drive as shown in Fig. 2. The experimental setup is composed of an AC servo motor with an encoder, a harmonic drive reducer and a high-precision encoder with resolution 18000. The high-precision encoder (HEIDENHAIN with a resolution of 18000 lines) is used to measure the actual output rotation of the harmonic drive, and the motor encoder (resolution of 8192 lines) is used to measure the actual input rotation of harmonic drive. Both the rotation signals are acquired simultaneously by using a public clock.

Experimental measurement. After the motor was controlled at low uniform velocity of $15(\mathrm{r} / \mathrm{min})$, the input and output rotations of integral circles were acquired and saved. Then the kinematic error was calculated according to Eq.1. The kinematic error result in respect of time is shown in Fig.3. As shown in Fig.3, the kinematic error showed slight beat frequency and obvious harmonic characteristics. Kinematic error measurements with different rotational velocities and directions were also executed. And Fast Fourier transform (FFT) of these kinematic errors were calculated. The peak to peak value and the frequency $a$ (with the largest FFT amplitude peak value) and frequency $b$ (with the second largest FFT amplitude peak value) were used to evaluate the kinematic error as shown in Table 1. From inspection of the table, there are slight changes in the kinematic errors with different velocities which may be caused by the elastic-dependent component in harmonic drive or measurement error. And the frequencies of all the kinematic errors with different velocities are basically twice and four times as large as the motor rotational frequencies. These observations will be helpful to the modeling of kinematic error. Further discussion about the constitution of kinematic error and the model method will be executed in next section. 


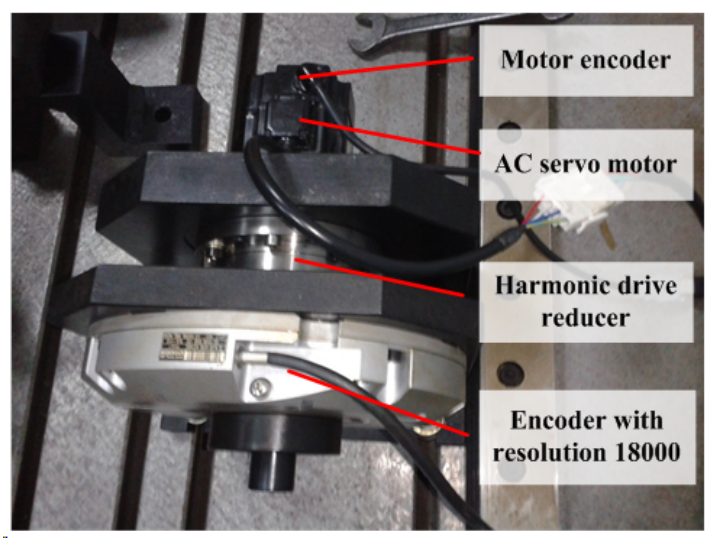

Fig.2 Experimental setup of kinematic error measurement.

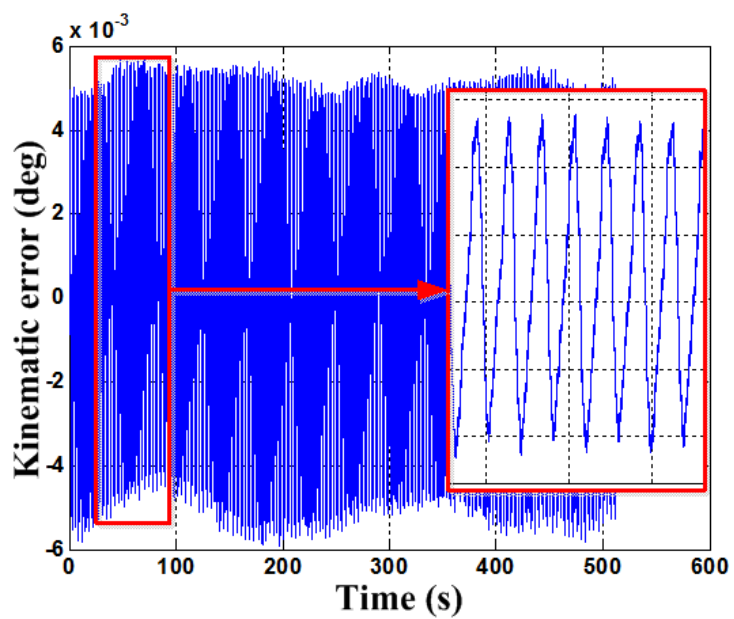

Fig.3 Measurement result of kinematic error.

Table 1 kinematic error with different velocities

\begin{tabular}{|l|l|l|l|}
\hline \multirow{2}{*}{$\begin{array}{c}\text { Velocity } \\
{[\mathrm{r} / \mathrm{min}]}\end{array}$} & \multicolumn{3}{|c|}{ Kinematic error } \\
\cline { 2 - 4 } & $\begin{array}{c}\text { Peak to peak } \\
\text { value }[\mathrm{deg}]\end{array}$ & Frequency $a[\mathrm{~Hz}]$ & Frequency $b[\mathrm{~Hz}]$ \\
\hline 6 & 0.0107 & 0.2035 & 0.4078 \\
\hline 12 & 0.0103 & 0.4061 & 0.8122 \\
\hline 30 & 0.0105 & 1.0160 & 2.0320 \\
\hline 60 & 0.0098 & 2.0240 & 4.0550 \\
\hline 120 & 0.0098 & 4.0060 & 7.9960 \\
\hline-6 & 0.0113 & 0.2042 & 0.4075 \\
\hline-12 & 0.0107 & 0.4067 & 0.8117 \\
\hline-30 & 0.0111 & 1.0150 & 2.0300 \\
\hline-60 & 0.0104 & 2.0350 & 4.0630 \\
\hline-120 & 0.0103 & 3.9930 & 8.0030 \\
\hline
\end{tabular}

\section{Kinematic error modeling}

As seen in the Fig.3, the kinematic error profile may be composed of a series of harmonics, then a fast Fourier transform (FFT) of the kinematic error at $15(\mathrm{r} / \mathrm{min})$ was calculated and the FFT data are shown in Fig. 4. The inspection of the spectral data reveals that the frequencies with significant peaks are $0.0039,0.5$ and $1 \mathrm{~Hz}$. 


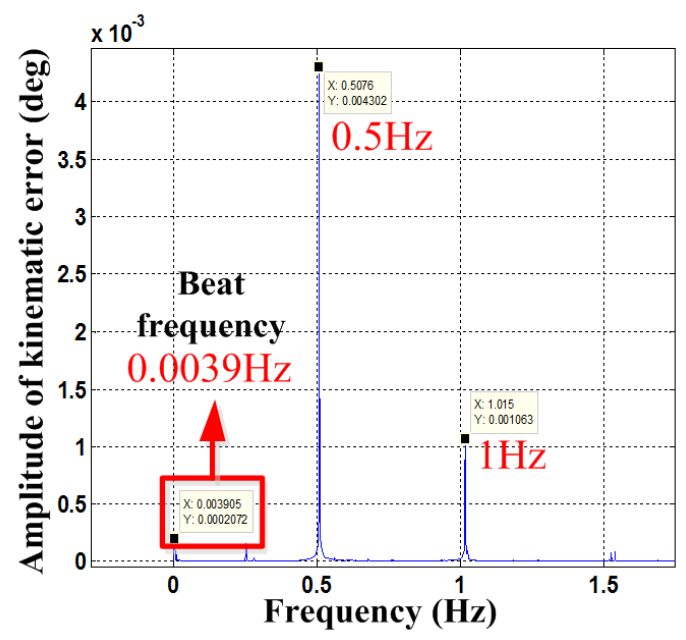

Fig.4 FFT result of kinematic error curve.

Beat frequency characteristic. Beat frequency characteristic is always caused by two harmonics with great close frequencies. In harmonic drive, there are two error components $e_{1}$ and $e_{2}$ whose frequencies are close. $e_{1}$ is the error component which changes with flexspline rotation, $e_{2}$ is the error component caused by misalignment of circular spline, and their frequencies are as follows respectively[8].

$$
\begin{aligned}
& f_{e_{1}}=2 \omega_{i} \frac{Z_{2}}{Z_{1}} . \\
& f_{e_{2}}=2 \omega_{i} .
\end{aligned}
$$

where $\omega_{i}$ is the wave generator rotation frequency, $Z_{1}$ and $Z_{2}$ are number of flexspline teeth and circular spline teeth respectively. Because the number of harmonic drive teeth is very large, about $60 \sim 320$, and the difference of the number between flexspline and circular spline is only two teeth, $Z_{2} / Z_{1}$ is definitely close to one. So $f_{e_{1}}$ and $f_{e_{2}}$ are very close. The beat frequency is described as the discrepancy between the two frequencies as follows.

$$
f_{b}=f_{e_{1}}-f_{e_{2}} \text {. }
$$

As the wave generator rotation frequency $\omega_{i}$ in our experiment was $0.25 \mathrm{~Hz}$ (corresponding to $15 \mathrm{r} / \mathrm{min}$ ), number of flexspline teeth $Z_{1}$ is 240 and number of circular spline $Z_{2}$ is 242 , the beat frequency is equal to $0.00416 \mathrm{~Hz}$. The beat frequency in FFT result from the experimental measurement data is $0.003905 \mathrm{~Hz}$ as shown in Fig. 4. The experimental result verified the theoretical analysis.

Position dependent kinematic error model. Actually, the kinematic error changes with the input motor rotational position because it is caused by definite manufacturing or assembling error. So a position domain analysis method was used here to model the kinematic error. Firstly, the experimental kinematic error curve should be resampled with a sample interval of $0.036^{\circ}$ by high-precision cubic spline method. The position dependent experimental kinematic error curve and data after resampling are shown in Fig. 5. Then a fast Fourier transform of the position dependent kinematic error curve was calculated in respect of motor revolutions and the FFT result is shown in Fig. 6. Because the inspection of the spectral data shows that the position domain frequencies with significant peaks are integers such as $0,2,4$ and 8 cycles per motor revolution, Fourier series method is applied here to analytically express the kinematic error and the expression is as follows[5]: 


$$
\hat{\varphi}_{\text {sim }}=\frac{p_{0}}{2}+\sum_{n=1}^{k}\left[p_{n} \cos \left(\omega_{n} \varphi_{i}\right)+q_{n} \sin \left(\omega_{n} \varphi_{i}\right)\right]
$$

where $\hat{\varphi}_{\text {sim }}$ is the Fourier series expression of the simulated kinematic error model, $\omega_{n}$ is the angular frequency of the harmonic, $p_{0}, p_{n}$ and $q_{n}$ are the undetermined coefficients and they can be determined from the numerical integration of the position dependent kinematic error $\hat{\varphi}\left(\varphi_{i}\right)$ as follows.

$$
\begin{aligned}
& p_{0}=\frac{1}{2 \pi} \int_{0}^{2 \pi} \hat{\varphi}\left(\varphi_{i}\right) \mathrm{d} \varphi_{i} . \\
& p_{n}=\frac{1}{\pi} \int_{0}^{2 \pi} \hat{\varphi}\left(\varphi_{i}\right) \cos \left(\omega_{\mathrm{n}} \varphi_{i}\right) \mathrm{d} \varphi_{i} . \\
& q_{n}=\frac{1}{\pi} \int_{0}^{2 \pi} \hat{\varphi}\left(\varphi_{i}\right) \sin \left(\omega_{\mathrm{n}} \varphi_{i}\right) \mathrm{d} \varphi_{i} .
\end{aligned}
$$

The number $k$ of the Fourier terms is decided by the normalized mean square error (MSE) which is defined as follows [9]:

$$
\operatorname{MSE}\left(\hat{\varphi}_{\text {sim }}\right)=\frac{100}{N \sigma^{2}} \sum_{i=1}^{N}\left[\hat{\varphi}_{s i m}\left(\varphi_{i}\right)-\hat{\varphi}\left(\varphi_{i}\right)\right]^{2}
$$

where $N$ is the number of the selected experimental data, $\sigma^{2}$ is the kinematic error variance. Generally, an MSE of less than 5.0 indicates a good fit.

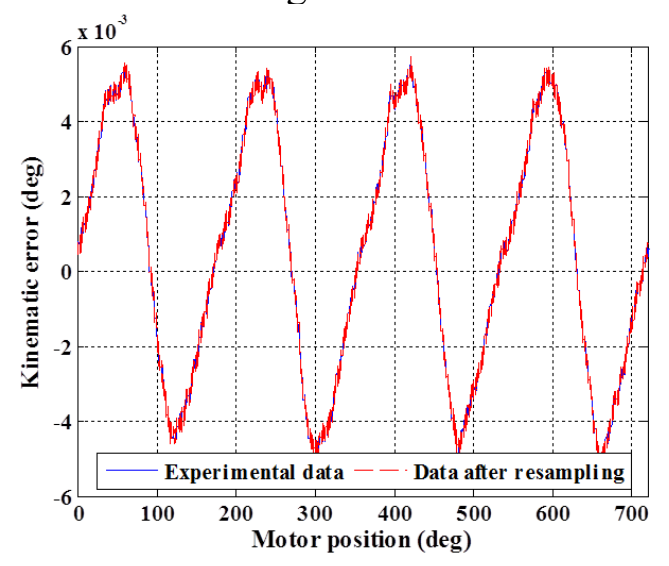

Fig.5 Position dependent kinematic error curve.

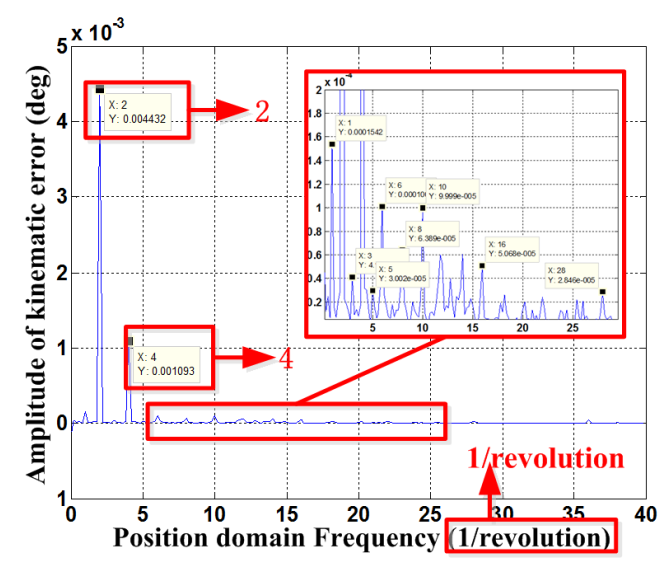

Fig.6 FFT result of position dependent kinematic error curve. 
Coefficients calculation and model verification. Among all the harmonic components, thirteen components with largest amplitude are used to approximate the kinematic error. In these components, the components with angular frequencies of 12, 14, 36 will cause bad MSE results, so other ten components are selected to establish the kinematic error model $\hat{\varphi}_{\text {sim }}$ and their frequencies and coefficients are displayed in Table 2 in descending order of their corresponding amplitude in FFT. When the model performance was evaluated by MSE, the experimental data points which were used to train the coefficients in Fourier expansion was compared with the simulated data firstly, then the experimental data points for another motor revolution was also used to compared with the simulated data and the MSE results corresponding to the number of Fourier terms $n$ are shown in Fig. 7. As seen in the figure, the sum of the first nine terms has a good MSE result of 0.49 which means an excellent fit and a model which has enough accuracy is established. The comparisons between the simulated data composed of the first nine terms and two sets of experimental data are shown in Fig. 8.

Table 2 Frequencies and coefficients of the Fourier

\begin{tabular}{|c|c|c|c|c|c|c|c|c|c|c|c|}
\hline \multicolumn{2}{|c|}{ Fourier terms $n$} & 1 & 2 & 3 & 4 & 5 & 6 & 7 & 8 & 9 & 10 \\
\hline \multirow{3}{*}{$\begin{array}{c}\text { Fourier } \\
\text { parameter } \\
\mathrm{s}\end{array}$} & $\omega_{n}$ & 2 & 4 & 1 & 6 & 10 & 8 & 16 & 3 & 5 & 28 \\
\hline & $\begin{array}{c}a_{n} \\
{\left[10^{-3} \mathrm{deg}\right]}\end{array}$ & 0.2057 & 0.0110 & -0.0825 & -0.0453 & 0.0705 & 0.0169 & 0.0170 & 0.0404 & -0.0203 & -0.0105 \\
\hline & $\begin{array}{c}b n \\
{\left[10^{-3} \mathrm{deg}\right]}\end{array}$ & 4.5000 & -1.0000 & 0.1557 & -0.0013 & -0.0644 & -0.0664 & -0.0357 & 0.0095 & 0.0114 & -0.0004 \\
\hline
\end{tabular}

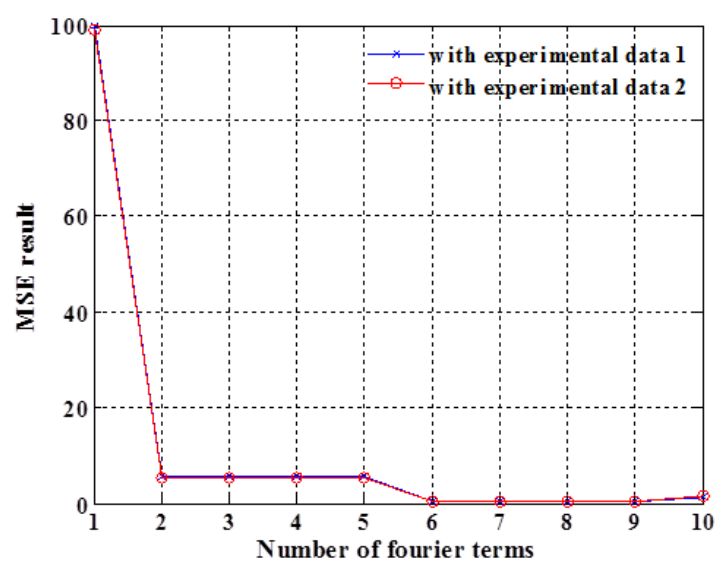

Fig. 7 MSE result between simulated data and experimental data

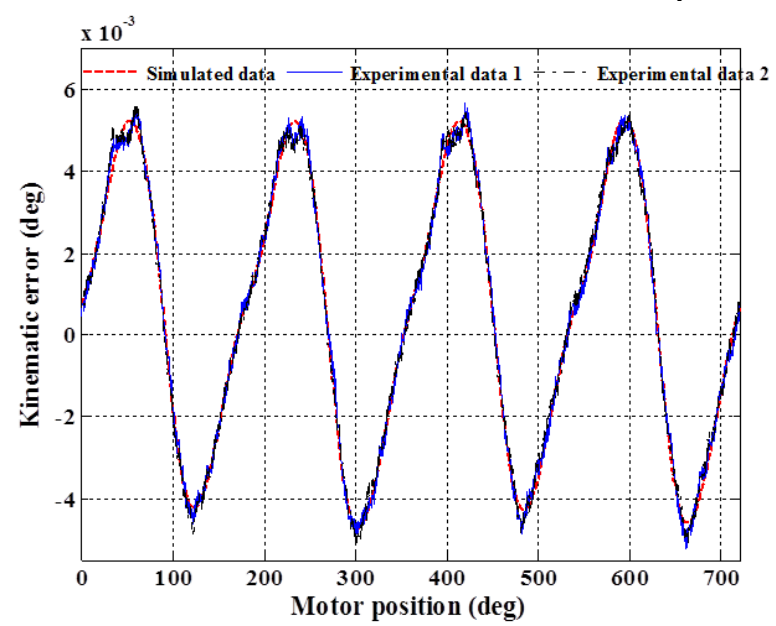

Fig. 8 Comparison between simulated data and experimental data

\section{Clearance experimental measurement}

The clearance in harmonic drive can be described as the output lag loss with the input reversing. It can be represented by the constant $\varphi_{c 0}$ as follows. 


$$
\varphi_{c}=\left\{\begin{array}{ll}
\varphi_{c 0} & \dot{\varphi}_{i}>0 \\
-\varphi_{c 0} & \dot{\varphi}_{i}<0
\end{array} .\right.
$$

The clearance was observed when the kinematic error was measured. If the input and output rotations were gathered before the motor rotated, the clearance would occur in the kinematic error curve. To pick up the clearance from the blended information, a reciprocating experiment was designed to measure and extract the clearance in harmonic drive.

Measurement. As shown in Fig 9, the motor drove the harmonic drive output to rotate for $72^{\circ}$ in positive direction and $36^{\circ}$ in negative direction and did this over and over again until $360^{\circ}$. The cycle time of the reversion in our experiment is $18 \mathrm{~s}$. Meanwhile, the input rotation and output rotation were acquired to calculate the kinematic error as shown in Fig. 10. And the clearance can be observed in this kinematic error curve as shown in enlarged drawing in Fig. 10.

Extraction of the clearance. As it is difficult to compare the positive and negative output rotation at one output position because of the kinematic error effect, the frequency domain method was used here to extract the clearance in harmonic drive. The FFT result without dc component is shown in Fig. 11. As shown in Fig. 11, there is an amplitude peak of frequency $0.053 \mathrm{~Hz}$ which is just corresponding to the reciprocal of reversion circle time 18s. So this amplitude can be regarded as the value of the clearance and this value is $0.0029^{\circ}$. The extraction result is just close to the value of $0.0022^{\circ}$ in technical manual [10] of the harmonic drive manufacturing company.
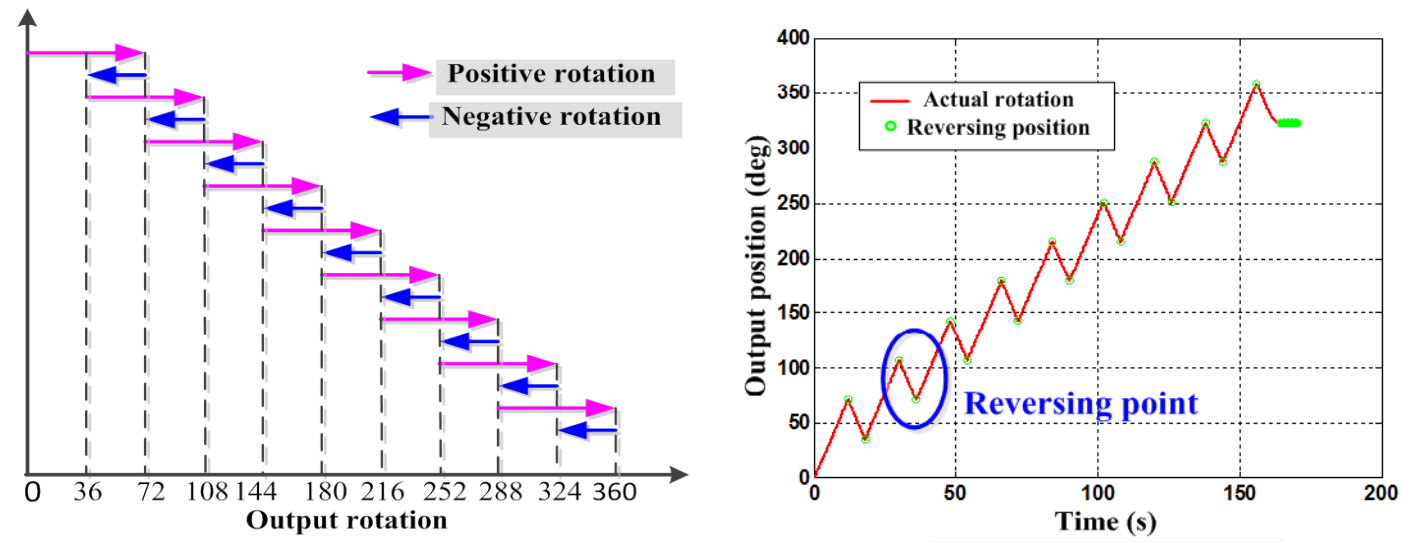

Fig. 9 Trajectory planning of clearance measurement

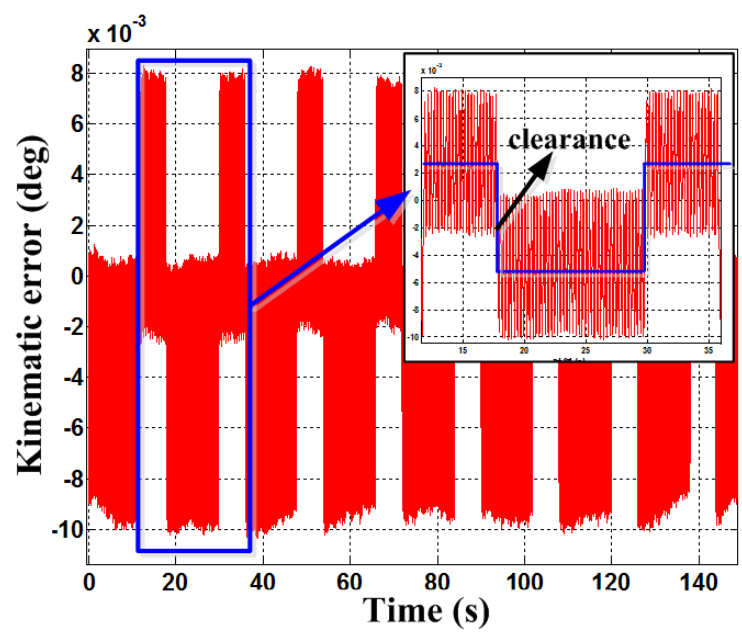

Fig. 10 Kinematic error including clearance 


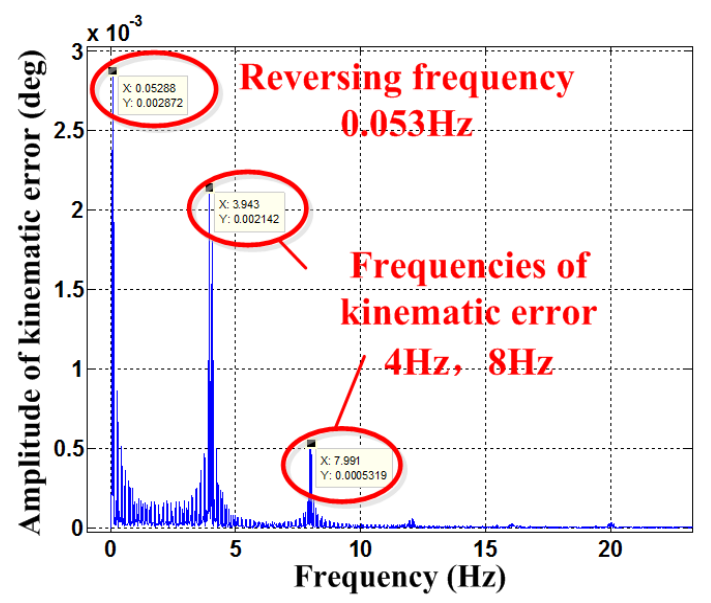

Fig. 11 FFT result of kinematic error including clearance

\section{Conclusion}

The experimental setup and measurement method proposed can be used to obtain the kinematic error and clearance in harmonic drive. The frequencies of the kinematic errors in harmonic drive are basically twice and four times as large as the input rotational frequencies. The Fourier expression can be used to model the position dependent kinematic error. And the beat frequency characteristic in harmonic drive is induced by two error components of the flexspline and circular spline. A reversing experiment can be applied to reveal the change process of clearance, and the clearance value can be extracted from the kinematic error by a frequency domain analysis method.

\section{Literature References}

[1] Rolf Slatter and Graham Mackrell. Harmonic Drives in Tune with Robots [J]. Industrial Robot, 1994, 21(3): 24-28.

[2] Good MC, Sweet LM and Strobel KL. Dynamic models for control system design of integrated robot and drive system. J Dyn Syst, Meas, Control 1985; 107(1): 53-59.

[3] Hidaka T, Ishida T, Zhang Y, et al. Vibration of a strain wave gearing in an industrial robot. In: Proc. of the 1990 International Power Transmission and Gearing Conference-New technology Power Transmission, New York, America, 1990, pp. 789-794.

[4] Tuttle TD and Seering WP. A nonlinear model of a harmonic drive gear transmission. IEEE Trans. Robot. Autom 1996; 12(3): 368-374.

[5] Ghorbel FH, Gandhi PS and Alpeter F. On the Kinematic Error in Harmonic Drive Gears. Transactions of the ASME 2001; 123(1): 90-97.

[6] Preissner C, Royston TJ and Shu D. A high-fidelity harmonic drive model. J Dyn Syst, Meas, Control 2012; 134(1): 1-13.

[7] Yamamoto $\mathrm{M}$, Iwasaki $\mathrm{M}$, Harai $\mathrm{H}$ and et al. Modeling and Compensation for Angular Transmission Error in Harmonic Drive Gearings. IEEJ Trans IA 2009; 139(1) 1-7

[8] Xiaochen S. Theoretical research and measurement on the transmission accuracy of the harmonic gearing transmission with new tooth profile. Thesis, Nanjing University of Science and Technology, Nanjing, 2014.

[9] Worden K, Wong CX, Parlitz $U$ and et al. Identification of pre-sliding and sliding friction dynamics Grey box and black-box models, Mechanical Systems and Signal Processing 2007; 21(1): 514-534.

[10]Harmonic Drive LLC, Comprehensive Dictionary of Harmonic Drive Combination Production, Harmonic Drive LLC, pp. 01-03, 2011. 